\title{
The Human Mandible: Lever, Link, or Both?
}

\author{
PHILIP D. GINGERICH \\ Museum of Paleontology, The University of Michigan, Ann Arbor, Michigan 48109
}

\author{
KEY WORDS Human anatomy . Jaw mechanics - Mastication
}

\begin{abstract}
Hylander (78) recently published important new data on bite force in humans, and showed that the human mandible cannot function purely as a link during incisal biting. He concluded instead that the mandible acts as a lever. Reexamination of Hylander's data suggests that the mandible cannot function purely as a lever either, and in fact it probably functions simultaneously as both lever and link during incisal biting.
\end{abstract}

Chewing in humans and other mammals is accomplished by a complex interaction of teeth, bones, and muscles. Dental and facial anatomists have long been interested in the human chewing mechanism. Some understanding of the mechanics of mastication is necessary in order to interpret evolutionary changes documented in the fossil record, thus anthropologists and paleontologists have also devoted much attention to this system and how it functions. Hylander (75) has reviewed the available literature and posed an important question in the title to his paper: "The human mandible: lever or link?" Subsequently, Hylander ('78) published new data bearing on this question and concluded that the mandible functions as a lever. However, further study of his data suggests that the human mandible probably functions simultaneously as both lever and link.

\section{LEVER OR LINK?}

Three forces are involved in the lever model of mandibular function: an applied force (muscle), a bite force along the tooth row, and a reaction force at the jaw joint(s). The bite force can never exceed the applied muscle force, and it is usually much smaller than the applied force because a large component is effectively wasted as reaction force. The link model of mandibular function is a special case of the lever hypothesis in the sense that the applied (muscle) and resultant (bite) forces are aligned. Hence there is no reaction force wasted at the jaw joint and the bite force is equal to the applied muscle force. In a previous paper, I emphasized the link model in interpreting the functional significance of translatory freedom in the mandible of humans and other vertebrates (Gingerich, '71). Hylander's (78) data are the first available that permit a test of the contribution of the link model to understanding mandibular function.

Humans, like most other mammals, have a complex masticatory system in which some muscles are aligned with the tooth row (middle and posterior temporalis), and others are not (anterior temporalis, superficial masseter, and medial pterygoid). Thus, the middle and posterior temporalis can potentially act directly to produce bite force by retracting the mandible, with the mandible functioning as a link. The anterior temporalis and the superficial masseter-pterygoid complex, on the other hand, are usually constrained to act by rotating the mandible about the condyle with the mandible functioning as a lever ... (an exception can occur when the bite is at the very back of the tooth row between aligned fibers of the masseter-pterygoid complex, a specialization of rodents and certain other mammals). During most biting the temporalis and masseter-pterygoid complex muscles act together, in which case the mandible could function simultaneously as a link with respect to the former and a lever with respect to the latter. An analysis such as that presented by Gingerich ('72: fig. 7) or its 3-dimensional generalization is necessary to resolve the bite force to muscle force to reaction force ratios when several different muscles with different alignments are firing simultaneously.

In favorable situations one can test whether the mandible is functioning as a link or as a lever by studying the direction of bite force in 


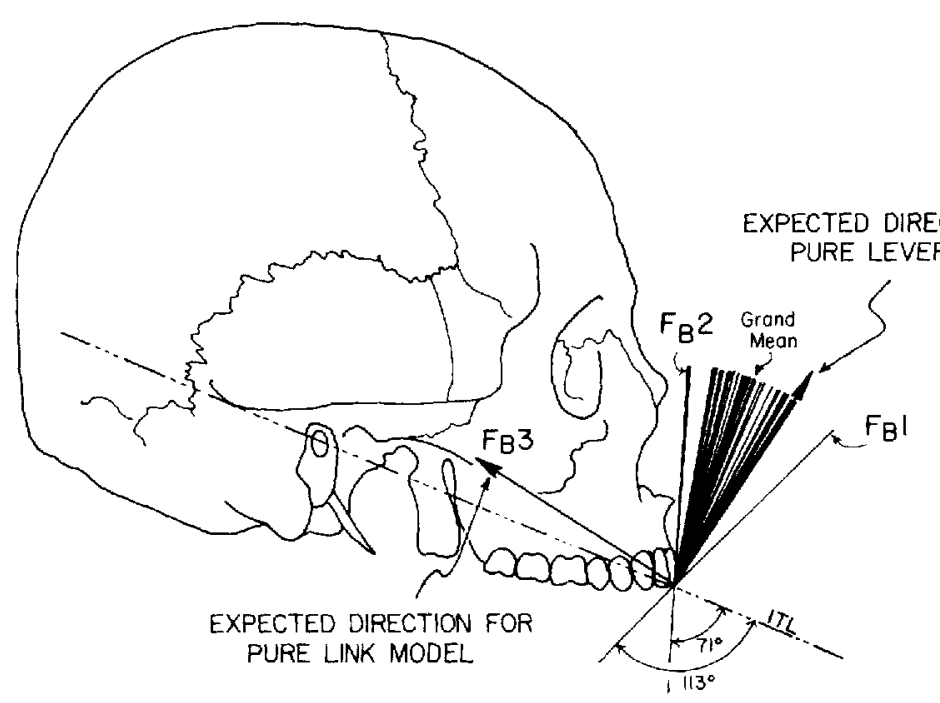

Fig. 1 Drawing of the lateral view of a human skull, illustrating the results of 30 experiments in incisal biting (after Hylander, '78: fig. 2). ITL is the incisor-tragus reference line. Large arrows represent expected directions of bite force if the mandible functions purely as a lever or purely as a link $\left(\mathrm{F}_{\mathrm{B}} 3\right)$ during incisal biting. Observed directions of bite force are 30 lines directed upward from the tip of the upper central incisor. Each line represents one experiment, which is averaged from five separate trials. Results range from $\mathrm{F}_{\mathrm{B}} 1$, at an angle of $113^{\circ}$ to ITL, down to $\mathrm{F}_{\mathrm{B}}$, at an angle of $71^{\circ}$ to ITL. The grand mean of all experiments is at an angle of $88.5^{\circ}$ to ITL, which is $12.5^{\circ}$ more posteriorly directed than expected if the mandible functions purely as a lever. The observed directions can be explained as a combination of both models, with the mandible func. tioning as both lever and link. Data from Hylander ("78: table 1).

actual subjects. Hylander ('78) designed an elegant series of experiments involving incisal biting in humans to test whether the mandible functions as a lever. During incisal biting, the direction of maximum bite force should be oriented in line with the direction of pull of the temporalis muscle if the mandible is functioning purely as a link, i.e., in the direction of $\mathrm{F}_{\mathrm{B}} 3$ in figure 2 of Hylander ('78) (see fig. 1 in this paper). If the mandible functions purely as a lever, the direction of maximum bite force should be directed as a tangent to the arc of mandibular rotation about the mandibular condyle. This tangent is drawn at the midpoint of the bite. For the 10-, 20- and $30-\mathrm{mm}$ biting blocks used in Hylander's experiments, this would be approximately perpendicular to the direction of $\mathrm{F}_{\mathrm{B}} 3$ in figure 2 of Hylander (78) (see fig. 1 in this paper).

Hylander (78) demonstrated that during incisal biting the mandible does not function purely as a link because bite force is oriented in a more orthal direction than would be expected under this hypothesis. He correctly concluded that the mandible must act as a lever. However, Hylander's data can also be used to test the hypothesis that the mandible functions purely as a lever during incisal biting. I have plotted the mean directions for Hylander's thirty experiments, taken from his table 1 (Hylander, '78). These observed values are shown graphically in figure 1 , where they are compared to bite directions expected if the mandible functions purely as a link or purely as a lever.

The expected direction of bite force if the mandible is functioning purely as a lever is at an angle of $101^{\circ}$, measured from the incisortragus line (ITL) as described by Hylander ('78). Examining Hylander's data, the mean for only one experiment significantly exceeds $101^{\circ}$ (this is $F_{B} 1=113^{\circ}$ in fig. 1). Three ex. periments yielded means of $100.5^{\circ}$ or $101.5^{\circ}$, the expected direction for the pure lever model. However, the remaining 26 experiments all yielded means falling below the expected value for a mandible functioning purely as a lever. The mean for all 30 experiments was $88.5^{\circ}$, which is $12.5^{\circ}$ less than predicted by the pure lever model. The observed values can only be explained as a combination of the two models, with the mandible functioning as 
both lever and link in the great majority of individuals. Hylander ('78) has shown that the lever component clearly predominates, but his data also indicate that there is a significant link component in mandibular function during incisal biting.

\section{DISCUSSION}

Human masticatory muscles can be divided broadly into two categories: (1) muscles that function parallel to the plane of sliding of the temporomandibular joint, with fibers aligned with the bite point (the middle and posterior fibers of the temporalis muscles are predominantly of this type), and (2) muscles that function perpendicular to the plane of sliding of the temporomandibular joint, with the fibers not aligned with the bite point (the superficial masseter and medial pterygoid muscles are predominantly of this type). Since the mandibular condyle is free to translate parallel to the line of action of muscles in category (1), the mandible functions mechanically as a link when these muscles contract (Gingerich, '71). At the same time, the mandibular condyle is not free to translate parallel to the line of action of muscles in category (2) because it abuts against the articular eminence of the squamosal, and the mandible functions as a lever with respect to these muscles. (Gingerich, '72: fig. 7).

In conclusion, it appears from Hylander's ('78) experiments that during incisal biting the human mandible functions as both a lever and a link simultaneously. It is clear that additional carefully controlled experiments like those published by Hylander ('78) will greatly increase our understanding of human mastication. This will in turn make possible a more complete interpretation of evolutionary changes in the human skull, mandible, and dentition.

\section{ACKNOWLEDGMENTS}

I would like to thank Doctor W. L. Hylander, Duke University, and Doctor D. S. Carlson, University of Michigan, for reading and im. proving the manuscript.

\section{LITERATURE CITED}

Gingerich, P. D. 1971 Functional significance of mandibular translation in vertebrate jaw mechanics. Postilla, 152: $1-10$.

1972 Molar occlusion and jaw mechanics of the Eocene primate Adapis. Am. J. Phys. Anthrop., 36: 359-368.

Hylander, W. L. 1975 The human mandible: lever or link? Am. J. Phys. Anthrop., 43: 227-242.

1978 Incisal bite force direction in humans and the functional significance of mammalian mandibular translation. Am. J. Phys. Anthrop., 48: 1-8. 